\title{
Synthesis and characterization of thermosensitive hydrogels with both supramolecular and hyperbranched structures
}

\author{
Ping Zhang, ${ }^{1,2}$ Qunfeng Liu, ${ }^{1,2}$ Yanxun Lan, ${ }^{1}$ Jianbo Shi, ${ }^{3}$ Mangeng $L u^{1 *}$ \\ ${ }^{1}$ Guangzhou Institute of Chemistry, Chinese Academy of Sciences, P. O. Box 1122, \\ Guangzhou 510650, People's Republic of China. E-mail: mglu@gic.ac.cn. Tel: 0086- \\ 20-85231089. Fax: 0086-20-85231089. \\ ${ }^{2}$ Graduate School of Chinese Academy of Science, Beijing 100049, People's \\ Republic of China. \\ ${ }^{3}$ Sun Yat-Sen University, Guangzhou 510275, People's Republic of China.
}

(Received: 20 May, 2007; published: 3 August, 2007)

\begin{abstract}
New novel kinds of thermosensitive hydrogels with supramolecular structures were synthesized by reversible addition fragmentation chain transfer (RAFT) polymerization of $N$-isopropylacrylamide (NIPAM) and polypseudo rotaxanes in the presence of $\mathrm{N}, \mathrm{N}$ - methylenebisacylamide (BIS) as a cross-linker. The polypseudorotaxanes were prepared by supramolecular self-assemblies of $\alpha$ cyclodextrins threaded onto (4A-PEG) polymers. The structure of the hydrogels were characterized in detail with FTIR techniques. The analytical results demonstrated that a channel-type crystalline structure of inclusion complexes (ICs) remains in as-obtained hydrogels. Their swelling behavior was measured gravimetrically in the temperature range from 20 to $60{ }^{\circ} \mathrm{C}$. The supramolecular structured hydrogels by RAFT polymerization (RAFT gels) showed accelerated shrinking kinetics to conventional hydrogel (CG), surprisingly.
\end{abstract}

\section{Introduction}

Recent advances in supramolecular chemistry are primarily associated with achievements in the study on self-assembly processes according to the guest-host type. Macrocyclic compounds are most often used as host molecules, among which cyclodextrins (CDs) have enjoyed the widest application [1]. CDs are a series of cyclic oligosaccharides consisting of six to eight glucose units, named $\alpha-, \beta-$, and $\gamma-$ $C D$, respectively, which possess internal hydrophobic cavities capable of accommodating various organic and polymeric compounds. Since the first report released in 1990 by Harada and his colleagues that $\alpha-C D$ can form inclusion complexes (ICs) with poly(ethylene glycol) (PEG) [2], wide variety of polymeric inclusion complexes based on CDs have been prepared and characterized [3-8]. Among the ICs ever reported, stimuli-responsive polyrotaxanes are of special interest. Many research groups have studied rotaxane-derived molecular shuttle in response to external stimuli, e.g., light, $\mathrm{pH}$, and polarity of the environment [9-14].

Stimuli-responsive polymers have been extensively investigated and used as smart biomaterials and drug-delivery systems [15]. The phase transition of polymers is induced by a continuous change in various conditions such as temperature [16], $\mathrm{pH}$ [17], electric field [18] or solvent composition [19]. 
Poly(N-isopropylacrylamide) (PNIPAM) gel is a widely studied, typical thermosensitive hydrogel, which displays phase transition as the temperature is increased above its lower critical solution temperature (LCST). However, it takes more than several hours to days for completion of volume shrinking, which is the main drawback for their practical usage, such as on-off valves and artificial muscles, and was an important topic to be solved. For this purpose, some successful strategies have been worked out. Comb-type grafted chains have been introduced to PNIPAM backbones and crosslinked for rapid deswelling. Okano's group [21] proposed a method for preparing comb-type PNIPAM hydrogels, which could collapse from a fully swollen state in less than $20 \mathrm{~min}$. They also reported comb-type grafted hydrogel composed of poly(ethylene oxide) (PEO) graft chains in the crosslinked PNIPAM network ${ }^{22}$. Lee et al. reported alginate/PNIPAM comb-type grafted hydrogels, which were able to respond rapidly to both temperature and $\mathrm{PH}$ changes [23]. We have synthesized PNIPAM-g-PNIPAM comb-type hydrogels by living radical polymerization technique [24]. Such hydrogels exhibited drastic acceleration of shrinking rate.

As known, micrometer-sized gel particle exhibited a more rapid response rate because of its small size and large surface area [25]. Some rapid responding bulk hydrogel system with microstructure has been reported. The formation of a porous structure has been shown to effectively enhance the deswelling rate of PNIPAM gels. The incorporation of surfactants [26], hydrogel preparation above its lower critical solution temperature (LCST) [27, 28], by a freeze dry [29], and the incorporation of silica microparticles removed by subsequent acid treatment of the silica [30], and by other means [31]; in all these cases, the deswelling rate of PNIPAM hydrogels has been enhanced by the formation of porous structures. In recent years, some other strategies were reported to improve the response rate. Hu et al synthesized a new class of nanostructured polymer gels by crosslinking gel nanoparticles through covalent bonds between functional groups on the surfaces of neighboring particles in solutions, which exhibited fast shrinking rate [32]. Cai et al have made fast responding bulk hydrogel by crosslinking poly(NIPAM-co-acrylic acid) microgels in a PNIPAM polymer network [33]. Zhuo et al reported fast-responsive bulky PNIPAM hydrogels by incorporating PNIPAM particles into PNIPAM networks to form composite hydrogels, which may be ascribed to the generation of pores that allow water molecules to be quickly squeezed out of the bulky PNIPAM gels [34]. Zhuo et al synthesized PNIPAM with water/acetone as a mixed solvent during the hydrogel crosslinking reaction to obtain a faster deswelling rate [35]. Carrying out the polymerization/ crosslinking of NIPAM in solid phase states also resulted in rapidly responsive PNIPAM hydrogels [36]. Zhuo et al also reported that the response rate of the PNIPAM hydrogel could be improved via incorporating siloxane linkage and so on [37].

As mentioned above, the synthesis of fast responsive hydrogels by new method has become an intriguing research area, motivated by both their theory and practical applications. In this study, new kinds of thermosensitive and supramolecular structured hydrogels were synthesized by RAFT polymerization of NIPAM and polypseudorotaxanes in the presence of BIS as a cross-linker. To our knowledge, there is only little report about the preparation of dissolvable chemical supramolecular structured hydrogels by RAFT polymerization. The swelling behavior was investigated and showed accelerated shrinking kinetics compared to conventional hydrogel, suggested a hyperbranched structure during gelation. 


\section{Results and Discussion}

\section{Preparation}

a-CDs appeared to act as a powerful gelator, and a rapid physical gelation occurred at room temperature upon a polymerizable macromer solution mixing with a aqueous solution of $\alpha$-CDs. The physical gels formed are thixotropic and reversible, and the sol-gel transition process can be readily controlled by adjusting the $\alpha-C D$ concentration and the composition of the diblock macromers. These gels were freeze dried to give ICs or polypseudorotaxanes.

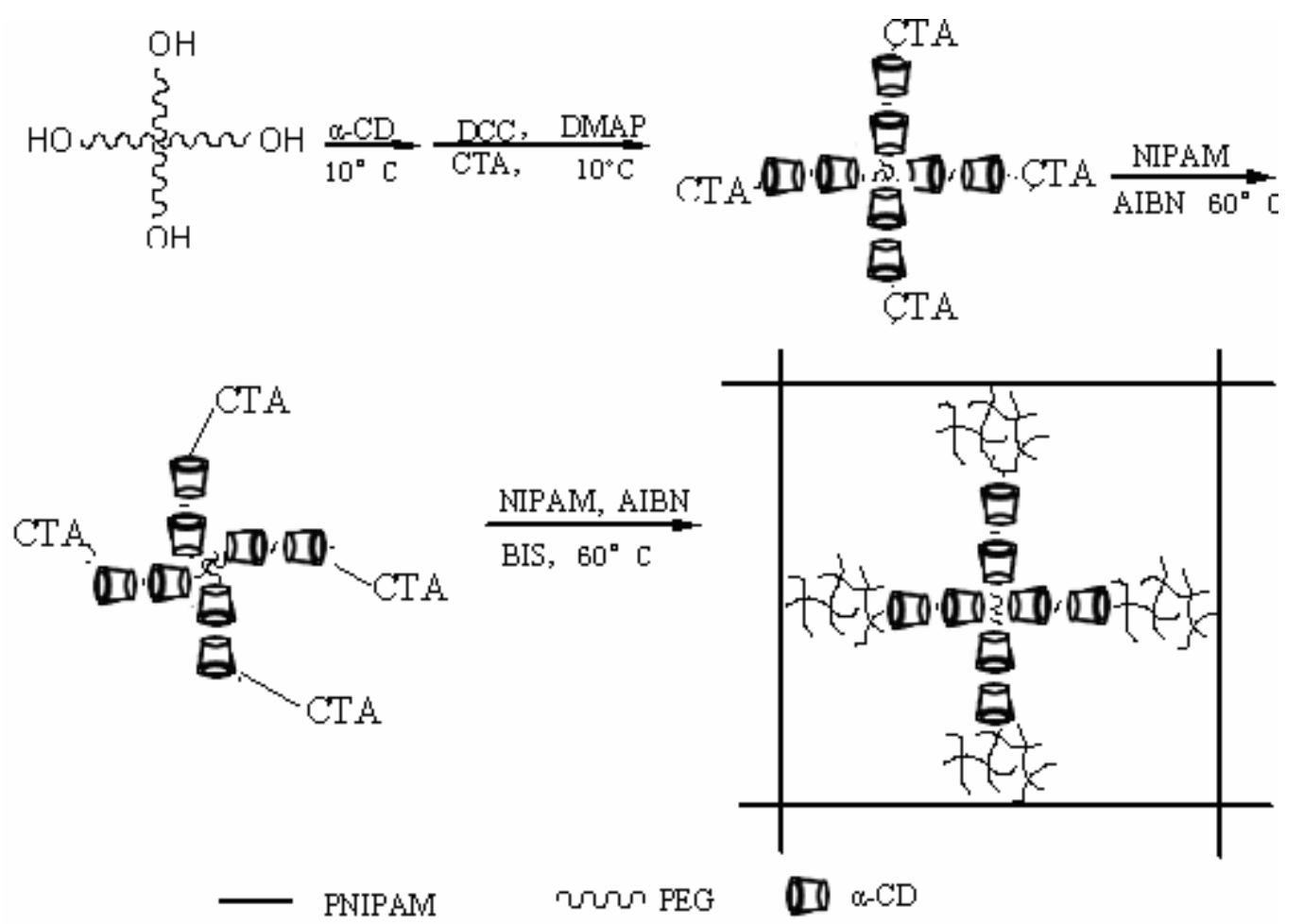

Fig. 1. Preparation route for thermosensitive and supramolecular structured hydrogels.

The preparation route of is described in Figure 1. In fact, the polymerization took place in the heterogeneous phase. So the dispersion of the monomer NIPAM in the aforementioned physical gels was a key step to ensure it being incorporated into the matrix chains. Therefore, sound stirring, sonication, and standing operations were prerequisites before RAFT polymerizing the mixture. A perfect suspension was formed after these physical gels mixed with the monomer NIPAM. When a perfect suspension was placed in oil at $60{ }^{\circ} \mathrm{C}$, a rapid polymerization in situ occurred in which the RAFT acid end groups and NIPAM were initiated and cross-linked to yield the dimensionally stable hydrogel. As shown in Table 2, the weight of all the hydrogel samples increases with the feeding molar ratio of $\alpha-C D$ to NIPAM, indicating the polymerization proceeded between the ICs or polypseudorotaxanes and NIPAM. Taken together the following structural characterization and the swelling examination, it is proper to prepare the thermosensitive and supramolecular structured hydrogels via this technique. In previous reports, Perrier et al synthesized PMMA hyperbranched polymers via the one-pot copolymerization of MMA and ethylene glycol dimethacrylate by RAFT polymerization [39]. The different polymeric 
architectures (gel and hyperbranched polymer) obtained by same method were ascribed to the different ratio of CTA/crosslinker. High CTA/ crosslinker ratio was unfavorable to the formation of gels because of CTA's retardation effect [40]. In our work, we find that the more the quality of diblock (ACT), the longer the crosslinking time. And when the feed ratio of diblock /BIS was over 0.5 , it failed to form gel. The phenomena indicate that the obtained hydrogels may exist as hyperbranched structure.

Conventional radical polymerization system

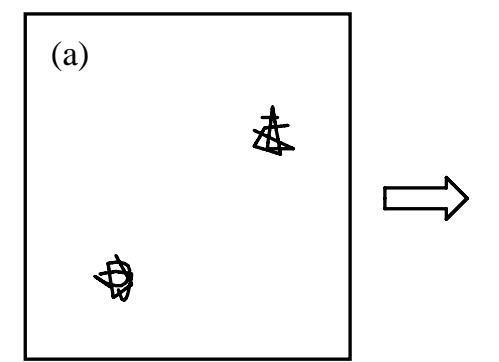

Intramolecular crosslinking

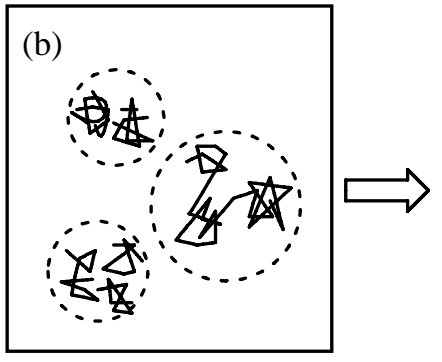

Microgel formation

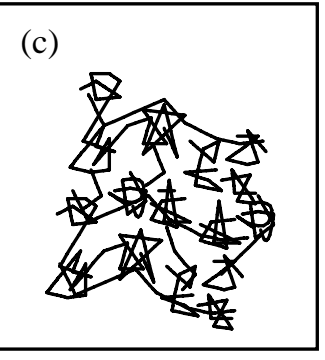

Gel

RAFT polymerization system

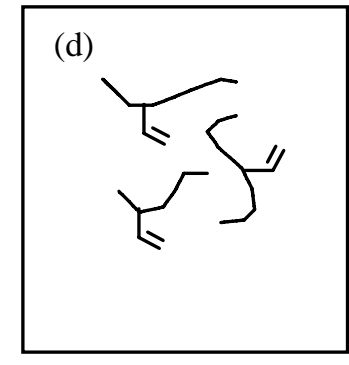

Linear

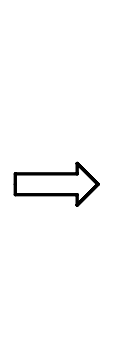

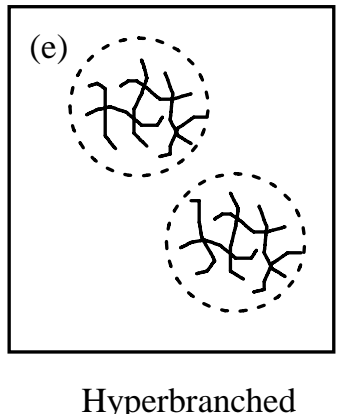

Hyperbranched

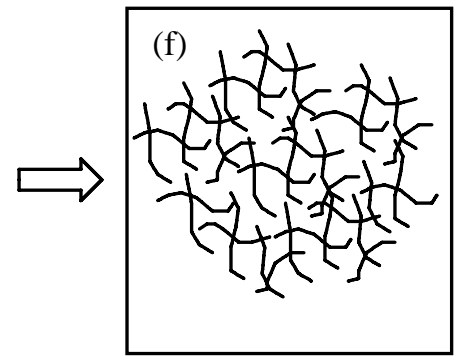

Gel

Fig. 2. Schematic presentations of the progression of gelation and different intrinsic structure of gels for $(a-c)$ conventional radical polymerization system (d-e) RAFT polymerization system

Matsumoto prepared amphiphilic network polymers by free radical crosslinking copolymerizations of monomethacrylate and dimethacrylate in the presence of lauryl mercaptan as a CTA, which led to the delayed gelation with a decrease in the primary polymer chain length. And the swelling ratios of the gels obtained were high [41]. It implied that the retardation effect might affect the structure and swelling property of the gels. We know that the increase of a polymer chain with thousands of monomeric units requires only seconds in a conventional free radical polymerization. However for RAFT polymerization the rate of polymerization decreases significantly when increasing the RAFT CTA concentration [42]. The distinction of rate might lead to different intrinsic structure of RAFT gels and GC. In the conventional system, (dead) polymers of full length are formed from the beginning of the reaction $[43,44]$. Then cross-links will be formed mostly within the same molecule, producing less expanded chains. As the reaction proceeds, intermolecular cross-linking will occur more frequently and combine these chains into larger molecules, which will absorb other chains more effectively than small molecules and leads to the generation of microgels even at relatively low conversions. At a higher (critical) conversion, these 
microgels will be tied up and form gel. But for RAFT system, the primary chains with living character form from the beginning of the reaction. Then living primary chains will further react with monomer and crosslinker to produce branched and hyperbranched polymers due to quite delayed gelation by the presence of CTA [39, 45]. When gelation occurs, some of the branched chains will remain in the network structure and lead to gels with a number of dangling chains [41]. Notably, the RAFT gels will be produced by random intermolecular crosslinking and the intramolecular cross-linking and microgel will be reduced greatly [43]. Figure 2 demonstrates the progression of gelation and the different intrinsic structure of gels by conventional radical copolymerization and by RAFT polymerization.

\section{Characterization}

FTIR spectra of hydrogel are presented in Figure 3 . The spectrum exhibits bands at 1103 and $1755 \mathrm{~cm}^{-1}$, which are attributed to the C-O-C stretching mode and the characteristic stretching vibration of carbonyl, respectively. Upon introducing PNIPAM segment, the the characteristic absorbance band of PNIPA is observed in the spectra. The amide I band ( $C=O$ stretch) emerges at $1651 \mathrm{~cm}^{-1}$, the amide II band $\left(\mathrm{N}-\mathrm{H}\right.$ vibration) at $1547 \mathrm{~cm}^{-1}$, and the methyl groups (in isopropyl group) at 1362-1386 $\mathrm{cm}^{-1}$. Moreover, the intensity of the amide I band increases as the NIPAM to the hydrogel. The $C=S$ groups was assigned to $1072 \mathrm{~cm}^{-1}$. The signal $1072 \mathrm{~cm}^{-1}$ was not obvious due to the low content of CTA in the NC Gels. The broad hydroxyl band is shifted to higher frequency at $3419 \mathrm{~cm}^{-1}$ owing to the formation of channel-type crystalline structure of ICs, which is most likely a consequence of the non-covalent interaction between $\mathrm{O}-\mathrm{H}$ of $\alpha-C D$ and $4 \mathrm{~A}-\mathrm{PEG}$ backbone.

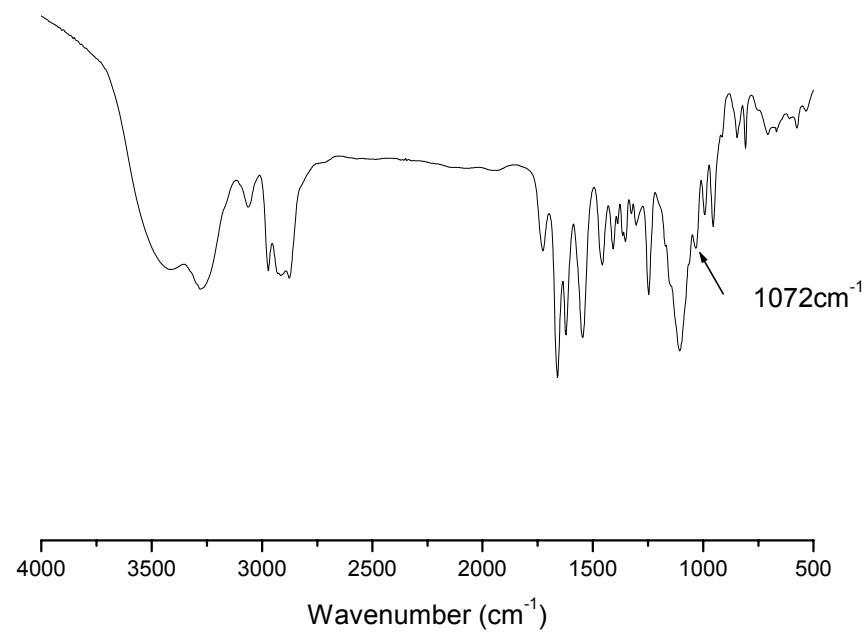

Fig. 3. FTIR spectra of supramolecular structured hydrogels.

Swelling and deswelling kinetics of hydrogels

According to feed compositions shown in Table 2, a series of hydrogels were prepared and their swelling ratios were measured in distilled water at $20^{\circ} \mathrm{C}$. The swelling ratios of hydrogels formed from diblock and polypseudorotaxanes with different amounts of $\alpha-C D s$ are presented in Figures 4, 5, 6, and 7 respectively. 
Compared with the CG, all the samples absorbed water quickly, and equilibrium water uptakes achieved after about 210 min for 1-sgel-1 and 300 min for 2-sgel-2 as shown in Figure 4 and 5.

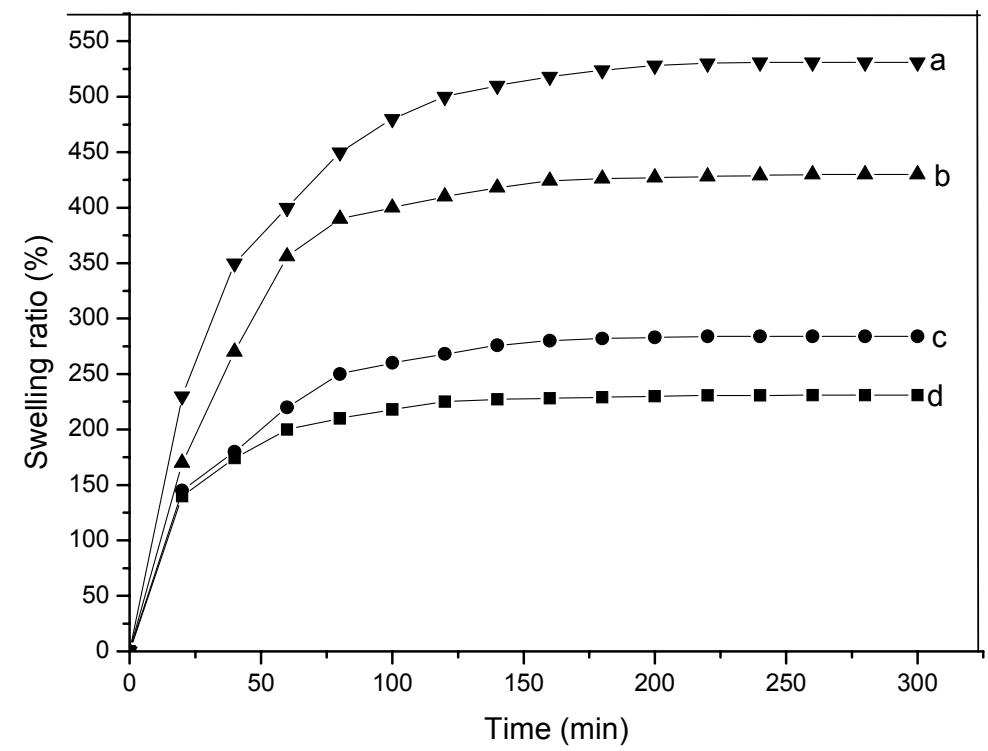

Fig. 4. Swelling ratio of a: 1-sgel-2, b: 1-sgel-3, c: 1-sgel-4, d: 1-sgel-5.

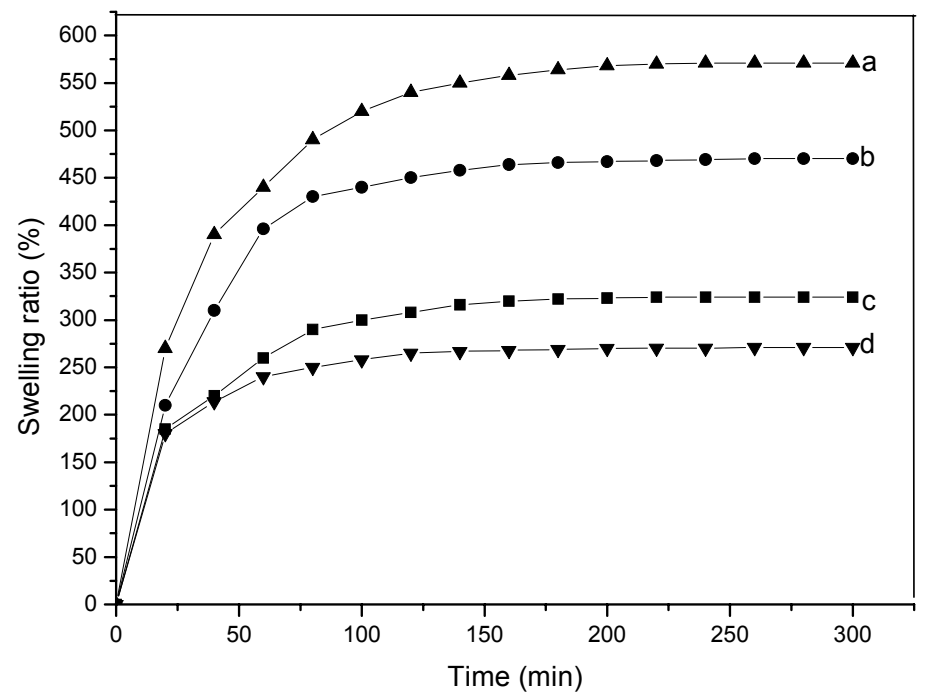

Fig. 5. Swelling ratio of a: 2-sgel-2, b: 2-sgel-3, c: 2-sgel-4, d: 2-sgel-5.

This result indicates that $4 A-P E G$ absorbed water can accelerate hydrogel backbone fast reaching swelling equilibrium. Incorporated $\alpha-C D s$ apparently decreased the swelling ratio of the hydrogels. Consequently, the equilibrium water uptakes decreased with an increase in the $\alpha-C D$ s threaded onto the gel network chains. A similar phenomenon was also observed for 2-sgel-2 with different amounts of $\alpha-C D s$. 
This result indicates that it is difficult for water to enter the matrix because of the rigidity of the network chains formed by the self-assemblies of $\alpha-C D s$. With diblock composition increased in the gel network chains, the swelling ratio of the hydrogels uptakes increased in Figure 6 and 7. In addition, with diblock composition increased in the gel network chains, the transparency of the hydrogels uptakes decreased, up to completely white. This result indicates that the hydrogels may exist as hyperbranched structure, which is consistent with our above mentioned previous report.

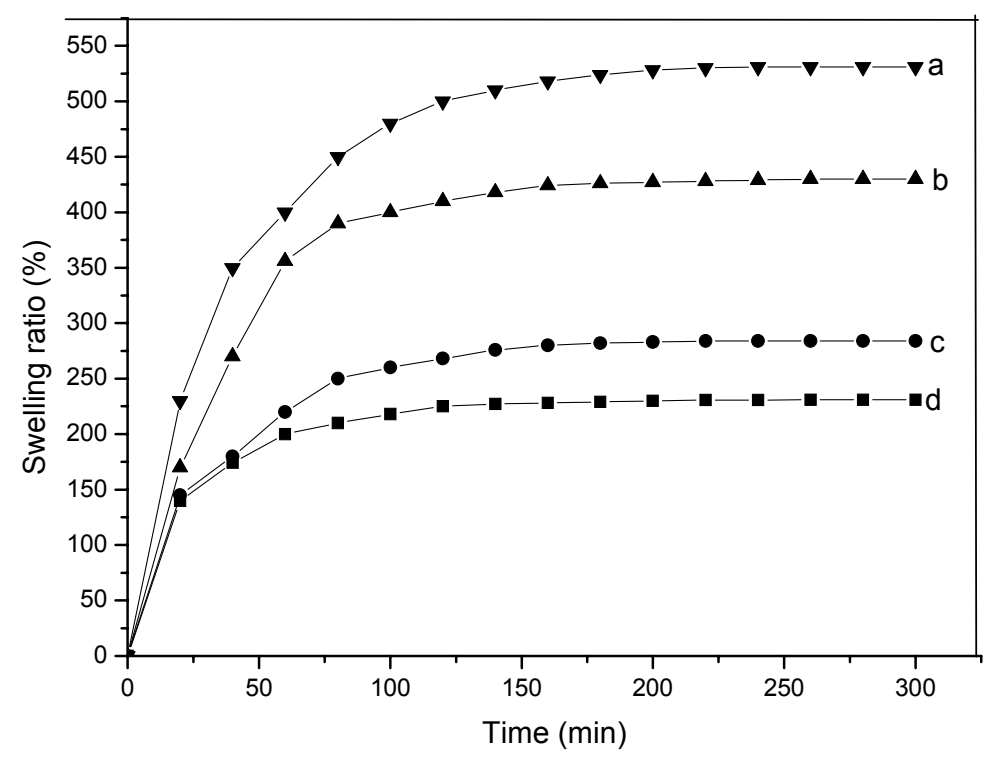

Fig. 6. Swelling ratio of a: 3-sgel-2, b: 3-sgel-3, c: 3-sgel-4, d: 3-sgel-5.

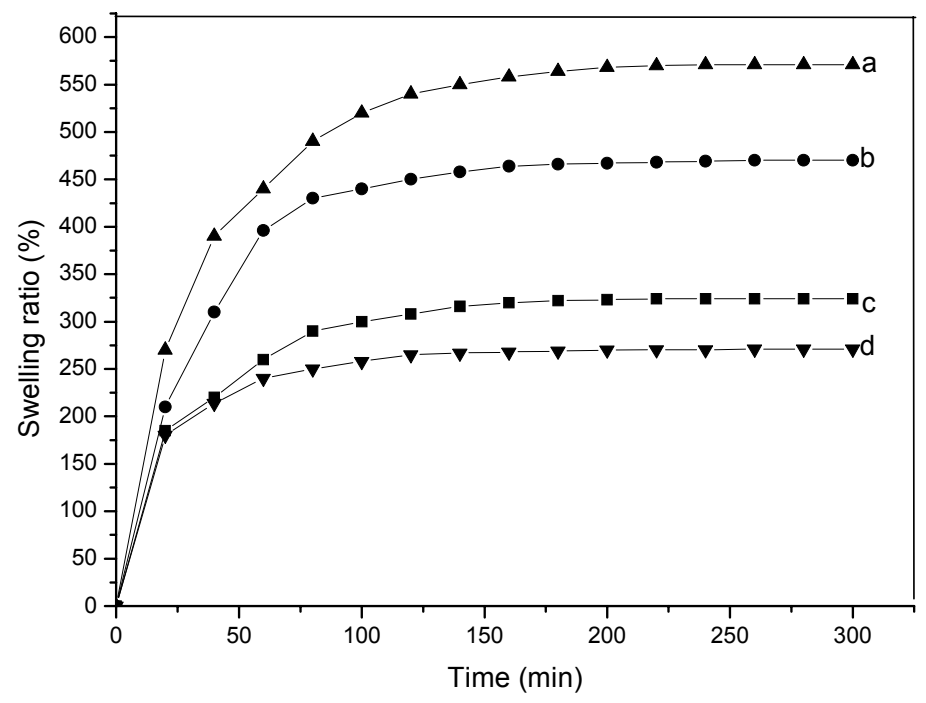

Fig. 7. Swelling ratio of a: 4-sgel-2, b: 4-sgel-3, c: 4-sgel-4, d: 4-sgel-5. 
Compared with the CG, RAFT gels showed accelerated shrinking kinetics. And the accelerated degree increased with the increased CTA/BIS ratio. All samples showed rapid deswelling kinetics. The results are consistent with our prediction. Figure 8 shows that the swelling degree is also directly correlated with the concentration of diblock used to prepare hydrogels.

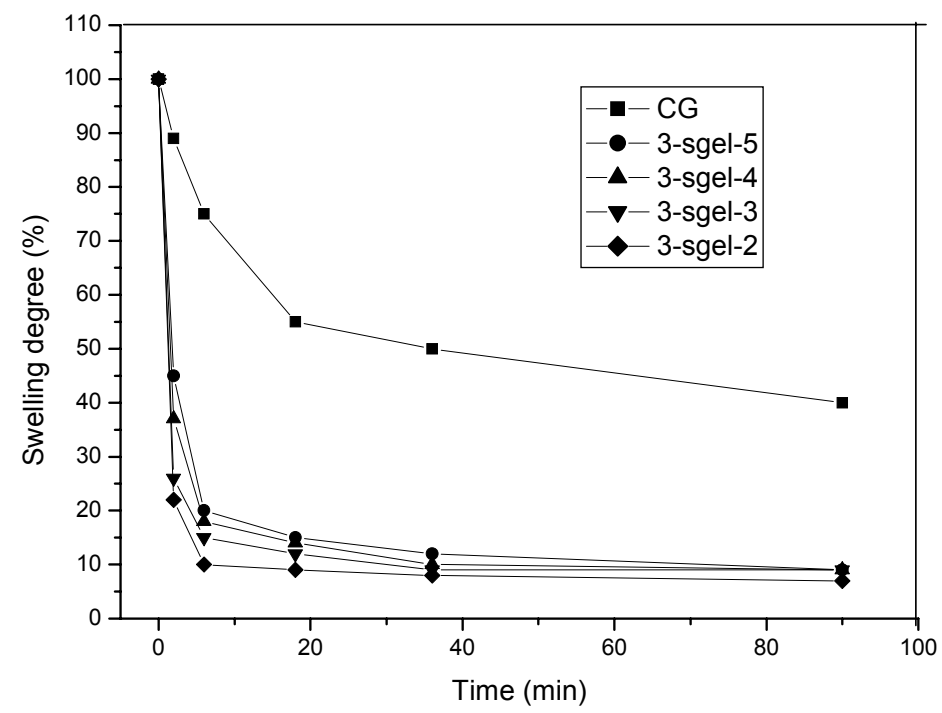

Fig. 8. Shrinking kinetics of conventional (CGel) and supramolecular structured hydrogels after T-jump from 20 to $50^{\circ} \mathrm{C}$.

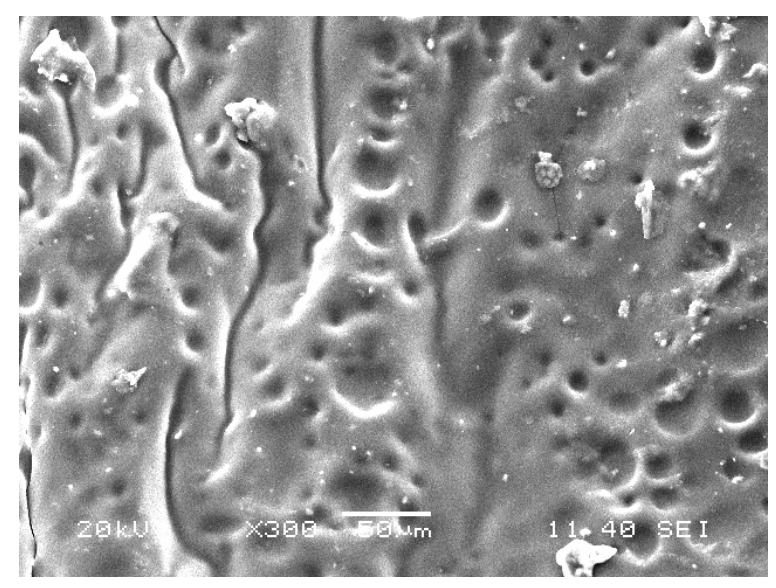

Fig. 9. The SEM photograph of the RAFT hydrogel.

When the NIPAM to 3-sgel series molar ratio was kept constant, the swelling degree of the hydrogel fell faster with the increasing composition of diblock. It was well known that the grafted gel would exhibit improved molecular mobility due to the existence of freely mobile graft chains, which show rapid dehydration, followed by the subsequent hydrophobic intermolecular aggregation of dehydrated graft chains. The dehydrated graft chains create the hydrophobic cores, which can enhance the hydrophobic aggregation of the networks, resulting in drastic acceleration of shrinking kinetics of grafted PNIPAM gel. So the RAFT gels exhibited acceleration of shrinking 
kinetics due to the presence of dangling chains. Among them, 3-Sgel-2 and 3-Sgel-3 show rapid shrinking rate indicating the RAFT gels with higher CTA/BIS rate have abundant dangling chains. Moreover $\alpha-C D$ s exist in the hydrogel matrix, which may induce the macroporous structure in the hydrogel network. The macroporous structure can accelerate response upon temperature. Figure 9 is the SEM photograph of the RAFT hydrogel. From Figure 9, we can see macroporous structure on the surface of the RAFT hydrogel clearly, which confirms our above supposition.

\section{Conclusions}

In this study, a novel type of thermosensitive hydrogel with both supramolecular and hyperbranched structures were synthesized via RAFT polymerization of NIPAM and diblock polypseudorotaxanes with BIS as cross-linkers. The supramolecular structure of inclusion complexes comprising a-CDs was found to remain in the hydrogels after polymerization. Their stimuli-responsive property can be tailored by changing the content of PNIPAM, the ratio of $\alpha-C D$ and diblock to macromer. It was found that the presence of CAT could lead to the branched structure and accelerated shrinking kinetics of RAFT hydrogels. The potential biomedical applications of these thermosensitive and supramolecular structured hydrogels in tissue engineered scaffolds, as biosensors in human body, and carriers of drug controlled release are now under investigation.

\section{Experimental}

\section{Materials}

2-Mercaptopropionic acid (99\%), carbon disulfide (99\%), N,N-methylenebisacyl amide (BIS), and benzyl bromide (98\%) were purchased from Aldrich and used without further purification. $N, N$-Dimethylacetamide $(99 \%)$ was stirred over calcium hydride for 2 days, distilled under reduced pressure, and stored under nitrogen. $\alpha$ Cyclodextrin (hydrate; Aldrich) was dried over $\mathrm{P}_{2} \mathrm{O}_{5}$ at $80^{\circ} \mathrm{C}$ under reduced pressure. Methanol was dried via refluxing with magnesium and subsequently distilled. Sodium acetate was dried for $12 \mathrm{~h}$ at $100{ }^{\circ} \mathrm{C}$ under reduced pressure. Four-arm poly(ethylene glycol) (4A-PEG; number-average molecular weight $=32,000 ; 10,020)$, N,N'dicyclohexylcarbodiimide (DCC), 4-(dimethylamino)pyridine (DMAP), and azo-bisisobutryonitrile (AIBN) were supplied by the Liming Chemical Engineering Research Institute (China). Synthesis of 3-Benzylsulfanylthiocarbonylsufanylpropionic Acid (RAFT Acid) was according to ref. 38.

\section{Preparation of polypseudorotaxanes}

A saturated aqueous solution containing a stoichiometric amount of $\alpha-C D s$ was added to a certain volume of $20 \%(\mathrm{w} / \mathrm{w}) 4 \mathrm{~A}-\mathrm{PEG}$ solution in water at $10{ }^{\circ} \mathrm{C}$. The resultant mixture was sonicated for $10 \mathrm{~min}$ and then stood for gelation. A gelation occurred to yield a physical gel network because of the supramolecular selfassemblies between $\alpha$-CDs and 4A-PEG. The gel formed was directly freeze-dried to give rise to polypseudorotaxanes.

\section{Preparation of RAFT acid-polypseudorotaxanes}

To synthesize RAFT acid-polypseudorotaxanes, a mixture of the polypseudorotaxanes (4A-PEG $\left.M n=10,020 / 32,000,2 * 10^{-4} \mathrm{~mol}\right)$, RAFT acid $\left(1 * 10^{-3}\right.$ 
mol), DCC $\left(2 * 10^{-3} \mathrm{~mol}\right), \operatorname{DMAP}\left(2^{*} 10^{-5} \mathrm{~mol}\right)$, DMF $(5 \mathrm{ml})$ were put into a glass tube, degassed and sealed under nitrogen. The reaction was stirred contiuously at $10{ }^{\circ} \mathrm{C}$ for one day. The reaction solution was filtered and then the final solution was precipitated in $30 \mathrm{ml}$ ether, filtered, and dried in vacuum at room temperature to constant weight; yield $80 \%$.

\section{Preparation of diblock PNIPAM-b-polypseudorotaxanes}

To synthesize the diblock PNIPAM-b-polypseudorotaxanes, a mixture of RAFT acidpolypseudorotaxanes, NIPAM, AIBN, and DMF were put into a glass tube, degassed and sealed under nitrogen. The polymerization was stirred continuously at $60^{\circ} \mathrm{C}$ for 10 hours. The reaction solution was precipitated in ether, filtered, and dried in vacuum at room temperature to constant weight. Results of the polymerization are summarized in Table 1.

Tab. 1. Feed composition for preparation of diblock PNIPAM-b-polypseudorotaxanes.

\begin{tabular}{ccccc}
\hline NIPAM & $\begin{array}{c}\text { RAFT acid- } \\
\text { Sample }\end{array}$ & (g) & AIBN & \\
polypseudorotaxanes $(\mathrm{g})$ & $(\mathrm{g})$ & DMF $(\mathrm{mL})$ \\
\hline Diblock-1 $^{\mathrm{a}}$ & 6.0023 & 5.0012 & 0.5021 & 25 \\
Diblock-2 $^{\mathrm{b}}$ & 3.2015 & 3.0005 & 0.3017 & 25 \\
\hline
\end{tabular}

a) PEG number-average molecular weight $=10,020$.

b) PEG number-average molecular weight $=32,000$.

Preparation of thermosensitive and supramolecular structured hydrogels using RAFT polymerization

To synthesize the hydrogels, a mixture of diblock PNIPAM-b-polypseudorotaxanes, NIPAM, BIS, AIBN, $\alpha-C D$, and DMF were put into a glass tube, degassed and sealed under nitrogen. After physical gels were formed, the polymerization was conducted at $60{ }^{\circ} \mathrm{C}$ under sound stirring, sonication, and standing operations. To remove unreacted monomer and linear polymers, the obtained gel was immersed in deionized water for 5 days at $5{ }^{\circ} \mathrm{C}$, and water was replaced every day. Then it was dried under ambient conditions for 24 hours followed by thorough drying under vacuum at $50{ }^{\circ} \mathrm{C}$ for 24 hours. Results of the polymerization are summarized in Table 2.

Tab. 2. Feed composition for preparation of thermosensitive and supramolecular structured hydrogels.

\begin{tabular}{ccccccc}
\hline Sample & $\begin{array}{c}\text { NIPAM } \\
(\mathrm{g})\end{array}$ & $\begin{array}{c}\text { Diblock-1 } \\
(\mathrm{g})\end{array}$ & BIS $(\mathrm{g})$ & AIBN $(\mathrm{g})$ & $\mathrm{DMF}(\mathrm{mL})$ & $\begin{array}{c}\text { Wt of gel } \\
(\mathrm{g})\end{array}$ \\
\hline 1-Sgel-1 & 0.7505 & 0.4015 & 0.0199 & 0.0615 & 4 & 0 \\
1-Sgel-2 & 0.7507 & 0.2009 & 0.0201 & 0.0610 & 4 & 0.9125 \\
1-Sgel-3 & 0.7503 & 0.2013 & 0.0204 & 0.0614 & 4 & 1.0947 \\
1-Sgel-4 & 0.7509 & 0.2007 & 0.0203 & 0.0613 & 4 & 1.1928 \\
1-Sgel-5 & 0.7504 & 0.2012 & 0.0205 & 0.0616 & 4 & 1.2937
\end{tabular}

a) $\alpha-C D$ concentrations in 1-Sgel-1, 1-Sgel-2, 1-Sgel-3, 1-Sgel-4, 1-Sgel-5 are $0.1018 \mathrm{~g}, 0 \mathrm{~g}, 0.1013 \mathrm{~g}$, $0.2009 \mathrm{~g}, 0.3007 \mathrm{~g}$. 


\begin{tabular}{ccccccc}
\hline Sample & $\begin{array}{c}\text { NIPAM } \\
(\mathrm{g})\end{array}$ & $\begin{array}{c}\text { Diblock-2 } \\
(\mathrm{g})\end{array}$ & BIS $(\mathrm{g})$ & $\begin{array}{c}\text { AlBN } \\
(\mathrm{g})\end{array}$ & DMF $(\mathrm{mL})$ & $\begin{array}{c}\text { Wt of gel } \\
(\mathrm{g})\end{array}$ \\
\hline 2-Sgel-1 & 0.7508 & 0.9013 & 0.0209 & 0.0615 & 4 & 0 \\
2-Sgel-2 & 0.7510 & 0.5007 & 0.0202 & 0.0610 & 4 & 1.2181 \\
2-Sgel-3 & 0.7505 & 0.5006 & 0.0207 & 0.0614 & 4 & 1.3984 \\
2-Sgel-4 & 0.7506 & 0.5008 & 0.0203 & 0.0613 & 4 & 1.4955 \\
2-Sgel-5 & 0.7502 & 0.5011 & 0.0211 & 0.0616 & 4 & 1.5921
\end{tabular}

b) $\alpha-C D$ concentrations in 1-Sgel-1, 1-Sgel-2, 1-Sgel-3, 1-Sgel-4, 1-Sgel-5 are 0.1003g, 0g, 0.1011g, $0.2008 \mathrm{~g}, 0.3004 \mathrm{~g}$.

\begin{tabular}{ccccccc}
\hline Sample & $\begin{array}{c}\text { NIPAM } \\
(\mathrm{g})\end{array}$ & $\begin{array}{c}\text { Diblock-1 } \\
(\mathrm{g})\end{array}$ & BIS $(\mathrm{g})$ & AIBN $(\mathrm{g})$ & $\mathrm{DMF}(\mathrm{mL})$ & $\begin{array}{c}\text { Wt of gel } \\
(\mathrm{g})\end{array}$ \\
\hline 3-Sgel-1 & 0.7505 & 0.4015 & 0.0199 & 0.0615 & 4 & 0 \\
3-Sgel-2 & 0.7510 & 0.3507 & 0.0209 & 0.0606 & 4 & 1.0157 \\
3-Sgel-3 & 0.7507 & 0.3011 & 0.0210 & 0.0611 & 4 & 1.1768 \\
3-Sgel-4 & 0.7505 & 0.2504 & 0.0204 & 0.0601 & 4 & 1.2336 \\
3-Sgel-5 & 0.7500 & 0.2012 & 0.0208 & 0.0609 & 4 & 1.2937
\end{tabular}

c) $\alpha$-CD concentrations in 1-Sgel-1, 1-Sgel-2, 1-Sgel-3, 1-Sgel-4, 1-Sgel-5 are $0.1018 \mathrm{~g}, 0 \mathrm{~g}, 0.1003 \mathrm{~g}$, $0.1001 \mathrm{~g}, 0.1005 \mathrm{~g}$.

\begin{tabular}{ccccccc}
\hline Sample & $\begin{array}{c}\text { NIPAM } \\
(\mathrm{g})\end{array}$ & $\begin{array}{c}\text { Diblock-2 } \\
(\mathrm{g})\end{array}$ & BIS $(\mathrm{g})$ & $\begin{array}{c}\text { AIBN } \\
(\mathrm{g})\end{array}$ & DMF $(\mathrm{mL})$ & $\begin{array}{c}\text { Wt of gel } \\
(\mathrm{g})\end{array}$ \\
\hline 4-Sgel-1 & 0.7508 & 0.9013 & 0.0209 & 0.0609 & 4 & 0 \\
4-Sgel-2 & 0.7505 & 0.8007 & 0.0213 & 0.0611 & 4 & 1.4995 \\
4-Sgel-3 & 0.7505 & 0.7007 & 0.0207 & 0.0603 & 4 & 1.6003 \\
4-Sgel-4 & 0.7506 & 0.6001 & 0.0203 & 0.0605 & 4 & 1.4909 \\
4-Sgel-5 & 0.7502 & 0.5011 & 0.0211 & 0.0612 & 4 & 1.4937 \\
\hline
\end{tabular}

d) $\alpha-C D$ concentrations in 1-Sgel-1, 1-Sgel-2, 1-Sgel-3, 1-Sgel-4, 1-Sgel-5 are $0.1003 \mathrm{~g}, 0 \mathrm{~g}, 0.1011 \mathrm{~g}$, $0.1003 \mathrm{~g}, 0.1015 \mathrm{~g}$.

The supramolecular self-assembly and gelation processes are illustrated in Figure 1. For comparison, CG was synthesized with NIPAM (0.4g), BIS (13mg) and AIBN (15mg) at $75^{\circ} \mathrm{C}$.

\section{Characterization}

FTIR spectra were measured using RFX-65A FTIR (Analect, America) spectrometer at room temperature in the range from 4000 to $500 \mathrm{~cm}-1$, with a resolution of $2 \mathrm{~cm}^{-1}$ and 20 scans. Samples were prepared by well dispersing the complexes in $\mathrm{KBr}$ and compressing the mixtures to form disks.

The swelling behavior of dried hydrogel was studied by a general gravimetric method. Dry films (a diameter of $10 \mathrm{~mm}$ and a thickness of $2 \mathrm{~mm}$ ) were incubated in distilled water at $20^{\circ} \mathrm{C}$, and the swollen weight for each sample was recorded at regular time intervals after excess surface water was blotted carefully with moistened filter paper. The procedure was repeated until there was no further weight increase. While the temperature increased gradually the swollen hydrogel began to shrink. The temperature was maintained constant for $3 \mathrm{~h}$ after increasing by every $5^{\circ} \mathrm{C}$, and then 
the weight of shrunk hydrogel was measured. The swelling ratio (SR) was calculated by the following equation:

$\mathrm{SR}=\left(m_{1}-m_{0}\right) * 100 \% / m_{0}$

where $m_{0}$ stands for the initial weight of dried gel and $m_{1}$ the weight of the swelling gel at a particular temperature and a prescribed time interval.

\section{References}

[1] Topchieva, I. N.; Tonelli, A. E.; Panova, I. G.; Matuchina, E. V.; Kalashnikov, F. A.; Gerasimov, V. I.; Rusa, C. C.; Rusa, M.; Hunt, M. A. Langmuir 2004, 20, 90369043.

[2] Harada, A.; Kamachi, M. Macromolecules 1990, 23, 2821-2823.

[3] Kawaguchi, Y.; Nishiyama, T.; Okada, M.; Kamachi, M.; Harada, A. Macromolecules 2000, 33, 4472-4478.

[4] Michishita, T.; Takashima, Y.; Harada, A. Macromol. Rapid Commun. 2004, 25, 1159-1162.

[5] Porbeni, F. E.; Edeki, E. M.; Shin, I. D.; Tonelli, A. E. Polymer 2001, 42, 69076912.

[6] Choi, H. S.; Ooya, T.; Sasaki, S.; Yui, N.; Ohya, Y.; Nakai, T.; Ouchi, T. Macromolecules 2003, 36, 9313-9318.

[7] Li, J.; Chen, B.; Wang, X.; Goh, S. H. Polymer 2004, 45, 1777- 1785.

[8] Li, J. Y.; Yan, D. Y. Macromolecules 2001, 34, 1542-1544.

[9] Ikeda, T.; Watabe, N.; Ooya, T.; Yui, N. Macromol. Chem. Phys. 2001, 202, 13381344.

[10] Nostro, P. L.; Lopes, J. R.; Cardelli, C. Langmuir 2001, 17, 4610-4615.

[11] Sun, G.; Wu, D.; Liu, Y.; He, C.; Chung, T. S.; Goh, S. H. Polymer 2005, 46, 3355-3362.

[12] Huh, K. M.; Ooya, T.; Sasaki, S.; Yui, N. Macromolecules 2001, 34, 2402-2404.

[13] Huh, K. M.; Tomita, H.; Ooya, T.; Lee, W. K.; Sasaki, S.; Yui, N. Macromolecules 2002, 35, 3775-3777.

[14] Fujita, H.; Ooya, T.; Yui, N. Macromol. Chem. Phys. 1999, 200, 706-713.

[15] Ding, Z.; Chen, Z.; Hoffman, A. S. Bioconjugate Chem. 1996, 7, 121.

[16] Tanaka, T. Phys. Rev. Lett. 1978, 40, 820.

[17] Hirokawa, Y.; Tanaka, T.; Katayama, S. Microbial Adhesion and Aggregation; Springer: Berlin, 1984.

[18] Tanaka, T.; Nishio, I.; Sun, S. T.; Ueno-Nishio, S. Science 1982, 218, 467.

[19] Katayama, Y.; Hirokawa, Y.; Tanaka, T. Macromolecules 1984, 17, 2642.

[20] Yoshida, R.; Uchida, K.; Sakal, K.; Kiruchi, A.; Sakurai, Y.; Okano, T. Nature 1995, 374: 2402.

[21] Kaneko, Y.; Nakamura, S.; Sakai, K.; Aoyagi, T.; Kikuchi, A.; Sakurai, Y.; Okano, T. Macromolecules 1998, 31: 6099.

[22] Ju, H. K.; Kim, S. Y.; Lee, Y. M. Polymer 2000, 42: 6851.

[23] Liu, Q.F.; Zhang, P.; Lu, M. G. J. Polym. Sci. Part A: Polym. Chem. 2005, 43:2615.

[24] Pelton, R. Adv. Colloid Interface Sci. 2000, 85:1.

[25] Antonietti, M.; Caruso, R. A.; Goltner, C. G..; Weissenberger, M. C Macromolecules 1999, 32:1383.

[26] Kabra, B. G.; Gehrke, S. H. Polym. Commun. 1991, 32, 322.

[27] Wu, X. S.; Hoffman, A. S.; Yager, P. J. Polym. Sci., Part A: Polym Chem 1992, $30: 2121$. 
[28] Kato, N.; Sakai, Y.; Shibata, S. Macromolecules 2003, 36:961.

[29] Serizawa, T.; Wakita, K.; Akashi, M. Macromolecules 2002, 35:10.

[30] Zhang, X. Z.; Yang, Y. Y.; Chung, T. S.; Ma, K. X. Langmuir 2001, 17:6094-9.

[31] Hu, Z.; Lu, X.; Gao, J.; Wang, C. Advanced Materials 2000,12:173.

[32] Cai, W. S.; Gupta, R.B. J. Appl. Polym. Sci. 2002, 83:169.

[33] Zhang, J. T.; Huang, S. W.; Xue, Y. N.; Zhuo, R. X. Macromol. Rapid. Commun. 2005, 26:1346.

[34] Zhang, X. Z; Zhuo, R. X.; Yang, Y. Y. Biomaterials 2002, 23:1313.

[35] Xue, W.; Hamley, I.; Huglin, M. B. Polymer 2002, 43:181.

[36] Zhang, X. Z; Zhuo, R. X. Langmuir 2001, 17:12.

[37] John, F. Q.; Rodney, P. C.; Thomas, P. D. J. Polym. Sci. Part A: Polym. Chem. 2002, 40:2956.

[38] Liu, B.; Kazlauciunas, A.; Guthrie, J. T.; Perrier, S. Macromolecules 2005, $38: 2131$.

[39] Monteiro, M. J.; Brouwer, H. Macromolecules 2001, 34:349.

[40] Perrier, S.; Barner-Kowollik, C.; Quinn, J. F.; Vana, P.; Davis, T. P. Macromolecules 2002, 35:8300.

[41] Ide, N.; Fukuda, T. Macromolecules 1997, 30:4268.

[42] Jiang, C. F.; Shen, Y. Q.; Zhu, S. P.; Hunkeler, D. J. Polym. Sci. Part A: Polym. Chem. 2001, 39:3780.

[43] Isaure, F.; Cormack, P. A. G; Sherrington, D. C. J. Mater. Chem. 2003, 13:2701. 